\title{
DEVELOPMENT OF AUTOMATIC GEO- FENCING AND ACCIDENTAL MONITORING SYSTEM BASED ON GPS TECHNOLOGY
}

\author{
Danish karim ${ }^{1}$ and Jaspal Singh ${ }^{2}$ \\ ${ }^{1}$ Department of ACSD, Centre for Development of Advanced Computing, Mohali \\ danishkarim04@gmail.com \\ ${ }^{2}$ Department of DESD Centre for Development of Advanced Computing, Mohali \\ jaspal_sng@yahoo.com
}

\begin{abstract}
The main objective of this project is to design and develop an embedded system for vehicle to detect the road accident for immediate medical help as well as for security purpose in theft prevention using single shock sensor. In case of accidental mode the system should inform automatically via text message to medical rescue team indicating the position of vehicle location. When there is no car driver, vehicle should automatically go into security mode. When a person tries to steal the vehicle, this system should send an alert message to the owner. The system Geo fencing and accidental monitoring system based on AT89S52 as a central core. This includes various technologies: a shock sensor, Liquid crystal display (LCD), Dual relay and wireless communication. This system also includes Geo fencing technology. Geo fencing enables the remote monitoring of predefined set of boundaries and automatic detect when vehicle exit from these areas.
\end{abstract}

\section{KEYWORDS}

Geo fencing, GSM, GPS, Shock Sensor

\section{INTRODUCTION}

The Vehicle accident is major public problem in many countries, particularly in India. This problem is rapidly increasing due to rider's poor behaviour such as speed driving, drunk driving, low use of seat belts and riding without sufficient sleep. India has the highest number of ranking due to road accident in the world according to world health organization (WHO) on the report of global status on road safety. In every hour, 40 people under the age of 25 die in road accident. According to WHO deaths due to road accident is the second most important cause of death for 5 to 29 year olds. According to National crime bureau (NCRB) the total death in India now crossed 135,000 mark due to road accident. Therefore, several research group and major vehicle manufacturing companies developed a safety device to protect riders from accidental injuries.

Alternatively an intelligent system is needed to detect accidents and safety of vehicle with tracking system to notify the accident to related people so that the quickest assistance can reach the people to save their life. The main reason behind this is to minimize the delay in medical facilities to reach the accidental place. This project insures the losses of life will be reduced rapidly. 
The system consists of cooperative components of shock sensor, microcontroller unit (MCU), GPS device to find the accident location and GSM module for sending the text message. Mobile text message is containing location from GPS (Latitude, Longitude) will be sent if vehicle accident is detected. A high performance 8 bit MCU AT89S52 is used to process and store real time signal from the shock sensor. This system also includes vehicle speed sensor (VSS) to monitor speed and location of vehicle by fleet operator.

\section{BACKGROUND}

\subsection{GPS TECHNOLOGY}

The Global Positioning System(GPS) is a system based on global navigation satellite system(GNSS) that provide reliable location and time information at all time in any whether condition on earth. It is composed of a network of 24 satellites of the united state which are previously used in military services, and later allowed for commercial use. The satellite emit radio signal of short pulses to GPS receiver periodically. A GPS receiver receives the signal from at least four satellites to compute its three dimension position that is latitude, longitude and altitude. Therefore GPS is a key technology for finding a device location.

\subsection{GSM MODEM}

A GSM MODEM is a special type of modem that accepts a SIM card and operates over a subscription to a mobile operator just like as a mobile phone. GSM modem is a wireless modem which sends and receives data through radio waves. A GSM modem requires a SIM card from a wireless carrier in order to operate Just like as a GSM mobile phone.

GSM modem support standard AT commands as well as extended set of AT commands. With the standard AT commands and extended AT commands, you can do things like:

- Sending SMS message

- Reading, Writing and Deleting SMS massage

- Monitoring the signal strength

- Reading, Writing and Searching phonebook entries

- Real time clock

\subsection{SHOCK/VIBRATION SENSOR}

A Shock sensor is capable of measuring shocks or vibration. When somebody or any obstacle hits the car, the sensor sends a signal indicating the intensity of motion. Depending upon the severity of intensity of shock, we use this sensor as for security purpose warning beep or sound or to monitor the accident for medical help. More advanced sensors send different information depending on how severe the shock is. The sensor to detect the vibration has a mechanical displacement to generate the alarm signal. There are two major detection methods to detect the vibration, one is mechanical detection. It works as a ON/OFF switch using the mechanical movement of metal of metal contact to detect true physical vibration. Other is acoustic sound detection may trigger false alarm by high noise car from the car. 


\section{DESCRIPTION OF SYSTEM}

Driving is an essential part of many people. Due to immense use of vehicles in day today life death due to accident has shown a tremendous growth rate. In most of the cases people loses their life due to delay in medical facilities to reach the accidental place. This project ensures to minimize the delay in medical facilities to reach the accidental place. This system includes communication system, positioning, sensing and other information related to technologies to improve the safety.

\subsection{Block Diagram}

The block diagram of this project is given in following figure. There are certain functions accomplished by these blocks. The descriptions of these blocks are as follows.

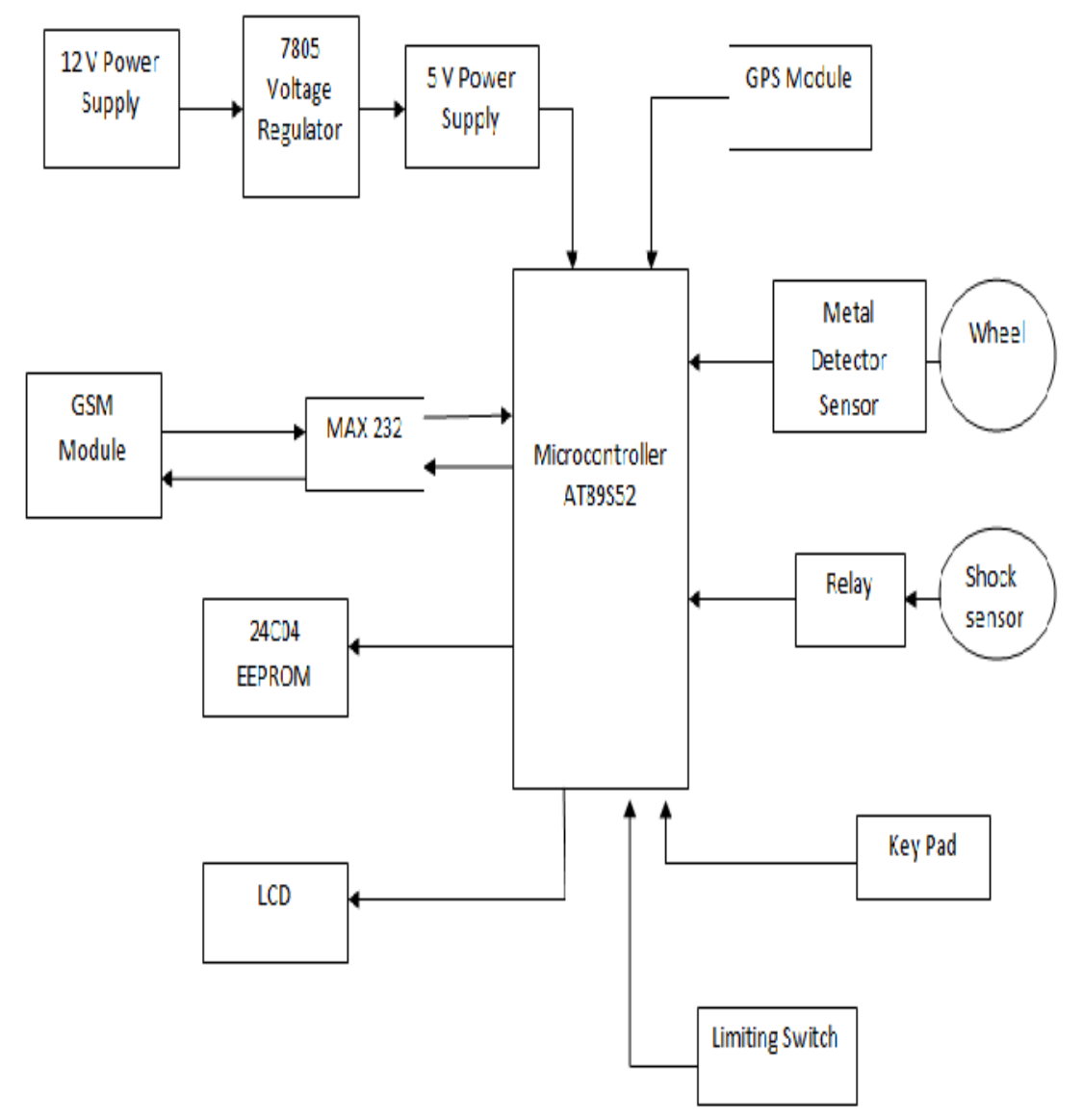

Figure 1- Block Diagram

\subsubsection{Power supply}

The power supply of our system is given by $12 \mathrm{~V}$ battery. Microcontroller unit is operated on $5 \mathrm{~V}$ supply. 5V supply is generated by using voltage regulator $7805.12 \mathrm{~V}$ is given to GPS module 
and GSM module. Basically the sensor module used in this project is shock sensor and Metal detector sensor. These both are needed to feed with power supply of $5 \mathrm{~V}$.

\subsubsection{Mode selecting switches}

Mode selecting switches are connected to the pins of microcontroller. Two switches are used as mode selection, in which this project works. There are two modes of operation, accidental mode and security mode. Whenever the driver is in the car accidental mode is selected. In this mode vibration is calculated by shock sensor and if this is greater than a threshold value say T2 then this sensor detect the accident. When there is no one on driving seat, the switch goes into the security mode to prevent thieving. A limiting switch is mounted car's driving seat. Limiting switch informs that the car driver is in the vehicle or not.

\subsubsection{Sensor module}

The sensor module is constructed with two sensors shock sensor and metal detector sensor. Metal detector sensor is mounted on wheel of the vehicle. A metal (iron in this case) is mounted at any place on wheel. Metal detector sensor senses metal and measures total number of revolution in a minute (rpm) and if this value is exceeded by certain limited value. The system will consider it of over speeding case. Shock senor is used to measure vibration or impact of collision to detect accident and send alert message with location if any accident happened.

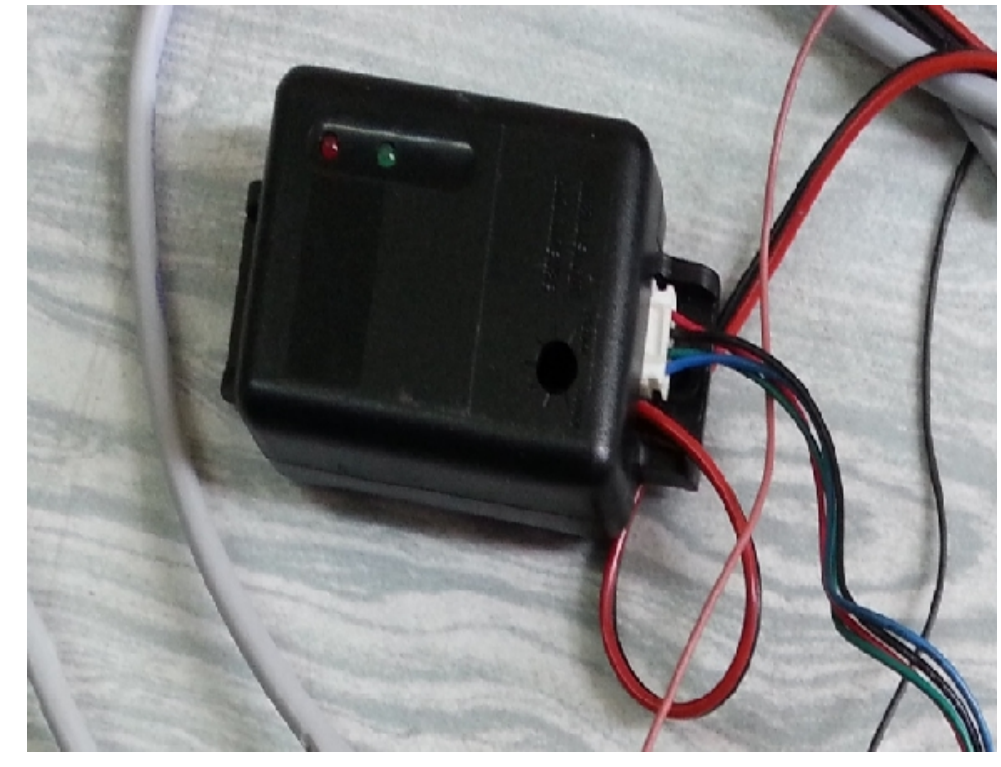

\subsubsection{Microcontroller unit}

Figure 2- Shock sensor

In microcontroller unit we use AT89S52 microcontroller. This is the main part of the project. Every command or instructions to the system is given by this unit. It receives the signals from sensor and GPS and sends message alert to medical rescue team or car's owner depending upon the mode of selection that is whether it is accidental or security mode. 


\subsubsection{EEPROM}

EEPROM is a non volatile memory to store small amount of data that must be saved when power is removed. In this project we use 24C04 EEPROM. The EEPROM saves numbers from which GSM module receives and sends text message. The saved number can also be edited through keypad. LCD displays the number which is stored in EEPROM. GSM module sends the data to saved number stored in EEPROM

All blocks in the diagram are implemented in the circuit level. The minimal circuit of system is shown in Figure 3

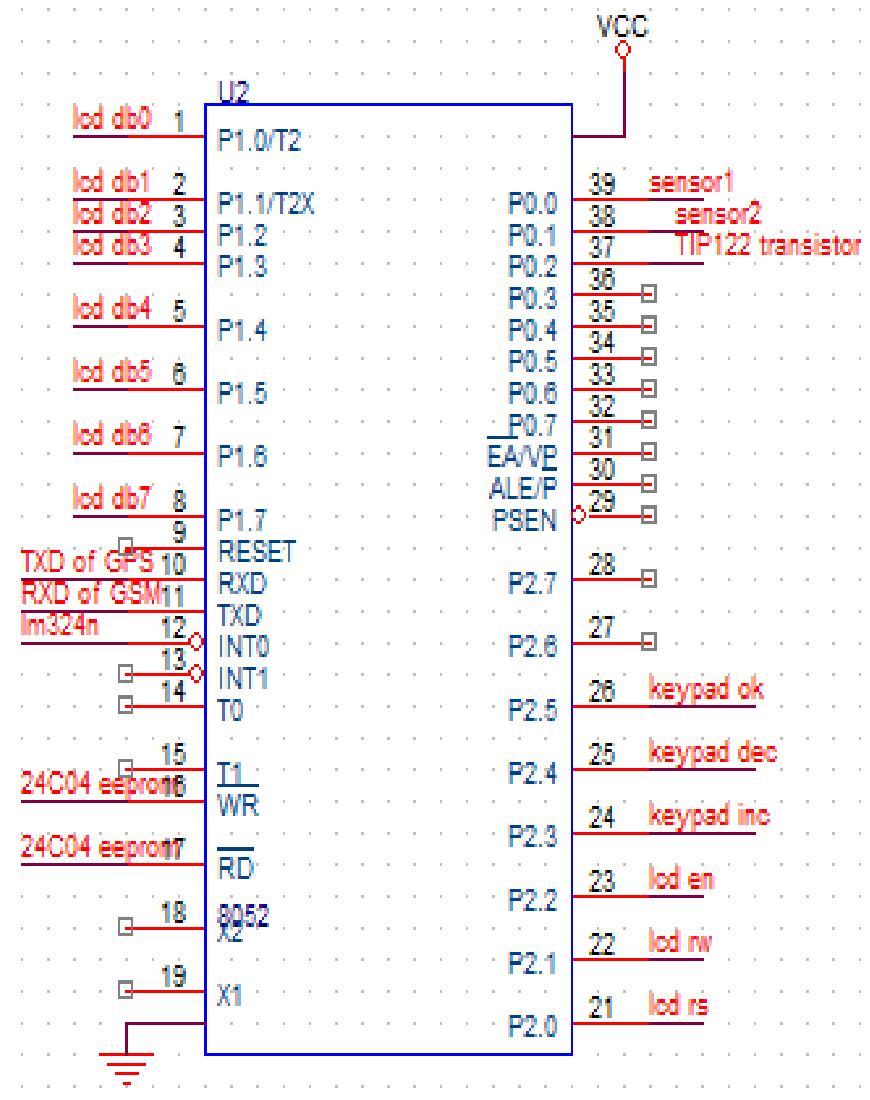

Figure 3: The minimal circuit diagram

\subsubsection{Keypad}

Keypad is used to save the 10 digit mobile number into EEPROM by pressing the keys. In this project we use only three keys named increment, decrement and ok to store number. Number saved into EEPROM is displayed on LCD.

\subsubsection{GPS module}

When the system is in accidental mode and if vehicle accident is detected, GPS device find the accident location and sent it to GSM module for sending the text message for immediate medical 
help. When the system is in either security mode or accidental mode and if fleet operator wants to know the vehicle location, GPS find the location and send it to GSM module.GSM module further send the text message containing vehicle location to the fleet operator.

\section{AUTOMATIC GEO FENCING ACCIDENTAL MONITORING SYSTEM METHODOLOGY}

Figure shows the software methodology of GPS based automatic geo fencing accidental monitoring system. Firstly, Software checks mode of selection whether the system is in security mode or accidental mode. Mode of selection can be found out by limiting switch which is mounted in car's driving seat. If limiting switch is not pressed, system automatically goes into security mode. In this mode shock sensor measure the vibration and this value is in between two predefined threshold value say T1 and T2 (T2>T1) then GSM module send alert message about theft prevention. In this mode fleet operator also want to know the vehicle location, then fleet operator sends a text message $* 0$ to find location of vehicle. In response system will send a text message containing vehicle location. Other mode of selection is accidental mode and if limiting switch mounted on car's driving seat is pressed then it goes into accidental mode. In accidental mode, vibration measured by shock sensor is compared with T2 and if this is greater than T2 system detect accident and system will send text message to medical help line number saved in EEPROM with accident location for immediate medical help. If there is accident detection, system continuously monitors speed of vehicle which is measured by vehicle speed sensor (VSS). Measured speed is compared with predefined threshold speed say S0.If speed measured by Vehicle speed sensor (VSS) is greater than S0 then system will send a text message about over speed to the Fleet operator. In this mode also fleet operator can also find out location of vehicle by sending text message $* 0$. In response the system will send text message to fleet operator containing vehicle location.

In this system the GSM module used is SIM900 which is based on AT commands as well as extended AT commands. Some of AT commands are shown table as follows:

\begin{tabular}{|c|c|}
\hline AT+CMGS & Send SMS Message \\
\hline AT+CMGR & Read SMS Message \\
\hline AT+CMGD & Delete SMS Message \\
\hline AT+CMGF & Select SMS Message Format \\
\hline AT+CMGW & Write SMS Message to Memory \\
\hline
\end{tabular}

Table 1: Various AT commands used in GSM Module 


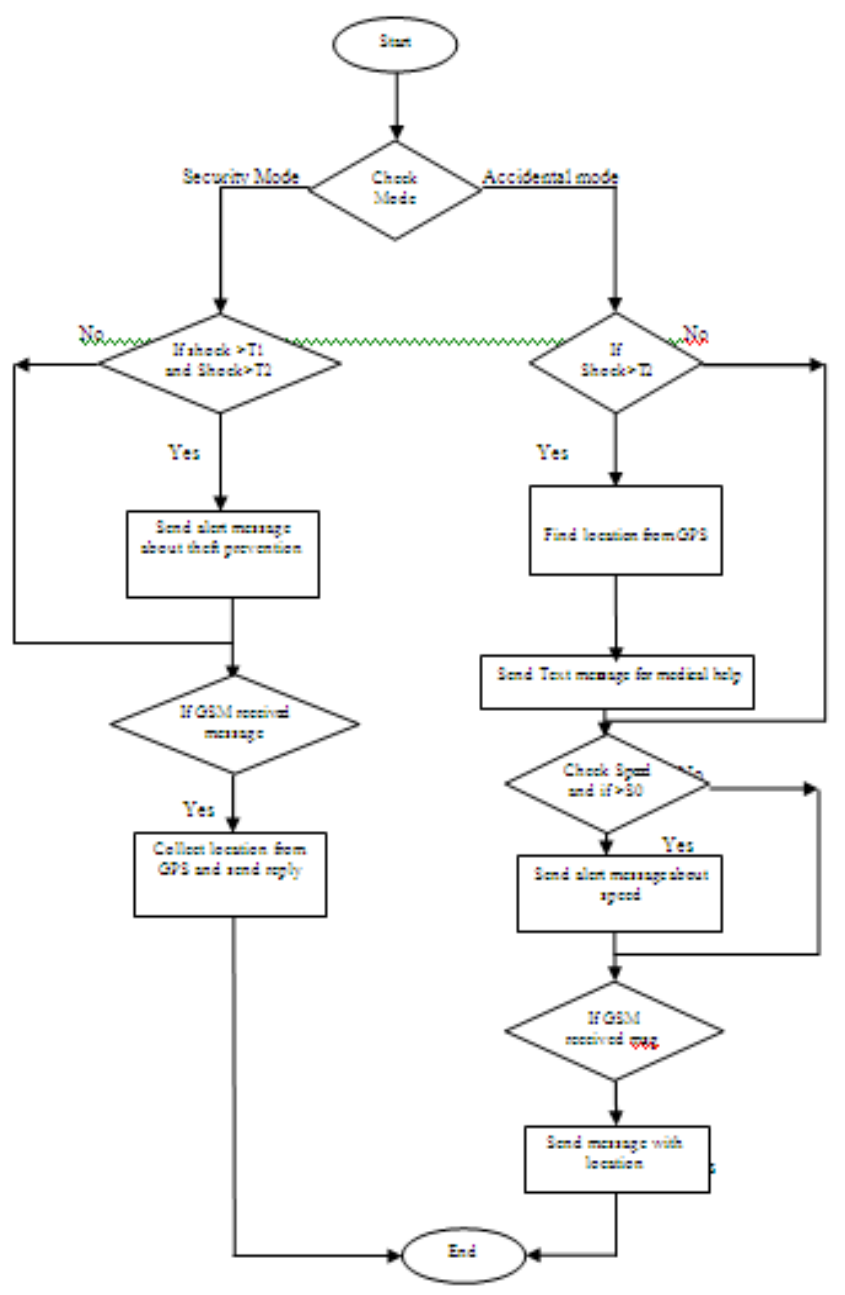

Figure 3: Program flow chart of monitoring system

\section{TESTING AND RESULTS}

Vibration based shock sensor, vehicle speed sensor (VSS), AT89S52, GPS and GSM modules are tested individually as well as an integrated system. The main advantage of shock sensor is that it measures small amount of vibration as well as large impact also. This is the reason that's why we use single shock sensor for both purposes. It works on the electromechanical principle. Shock sensor operates in accordance with electrodynamics principle and is used for measuring the bearing absolute vibration. This system also uses vehicle speed sensor to prevent over speeding and rough driving. This system send alert message to the fleet operator if any vehicle exceed its speed limit. This system also send a geo fencing alert that is any of these vehicles exit from a limited geographical area or geo fence area, an alert notification will be sent to the fleet operator. Tests are carried out on motor cycles and result is getting successfully. 


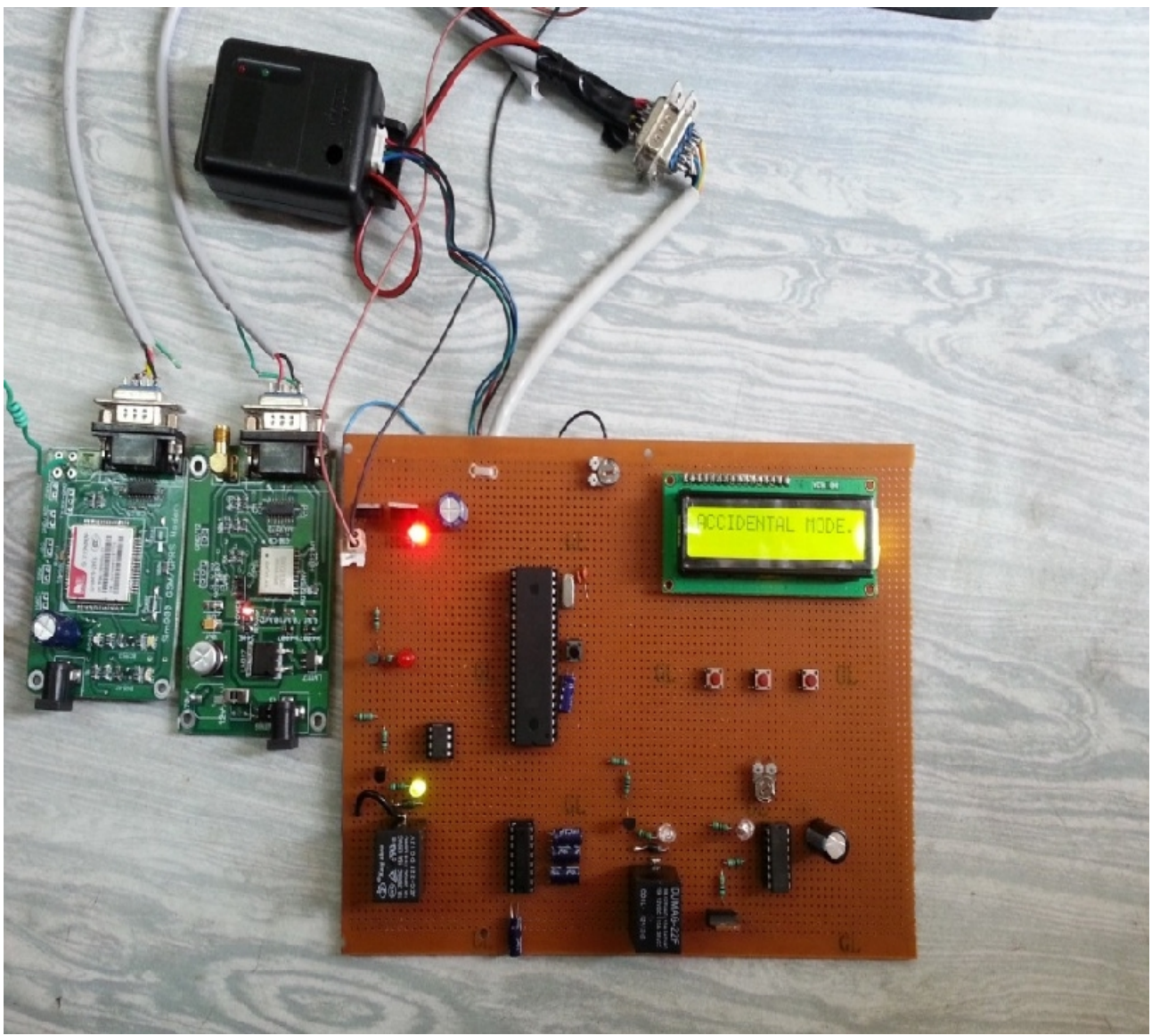

Figure 5- Testing Circuitry Board

\section{CONCLUSION}

In this paper, we proposed and described automatic geo fencing and accidental monitoring system based on GPS technology. Main advantage of this system is that during accident detection, it reduces the delay in medical facilities and prevents the losses of life. We are using single shock sensor for both purposes that is for accident detection and security purpose to prevent vehicle thieving. In this system we are using vehicle speed sensor of measuring speed to prevent rough driving and over speeding.

\section{ACKNOWLEDGEMENT}

We would like to extend a special thanks to C-DAC Mohali for providing us means to carry out our research work in precious way. We are also grateful to MHRD, Govt. of India for providing us platforms to our research work. 


\section{REFERENCES}

[1] Watthanawisuth, N.; Lomas, T.; Tuantranont, A., "Wireless black box using MEMS accelerometer and GPS tracking for accidental monitoring of vehicles," Biomedical and Health Informatics (BHI), 2012 IEEE-EMBS International Conference on , pp.847,850, 5-7 Jan. 2012

[2] VChadil, N.; Russameesawang, A.; Keeratiwintakorn, P., "Real-time tracking management system using GPS, GPRS and Google earth," Electrical Engineering/Electronics, Computer, Telecommunications and Information Technology, 2008. ECTI-CON 2008. 5th International Conference, pp.393,396, 14-17 May 2008

[3] In Jung Lee, "An accident detection system on highway using vehicle tracking trace," ICT Convergence (ICTC), 2011 International Conference , pp.716,721, 28-30 Sept. 2011

[4] Almomani, I.M.; Alkhalil, N.Y.; Ahmad, E.M.; Jodeh, R.M., "Ubiquitous GPS vehicle tracking and management system," Applied Electrical Engineering and Computing Technologies (AEECT), 2011 IEEE Jordan Conference, pp.1,6, 6-8 Dec. 2011

[5] Jog, S.R.; Sutaone, M.S.; Badawe, V.V., "Ruggedisation methodologies for GPS based Vehicle Tracking System," Electronics Computer Technology (ICECT), 2011 3rd International Conference on , vol.2, no., pp.214,218, 8-10 April 2011

[6] Jerath, K.; Brennan, S.N., "GPS-free terrain-based vehicle tracking on road networks," American Control Conference (ACC), 2012, pp.307,311, 27-29 June 2012

[7] Ravindra B. Sathe and A.S. Bhide"GPS-based soldier tracking and health monitoring system"World Journal of Science and Technology 2012, 2(4):97-99 ISSN: 2231 - 2587

[8] Neeti Bisht, Pragya Siddhi, Hema Kashyap "Monitoring Road Accidents using Sensors and providing Medical Facilities", 2012

[9] G. Gnanavel "Embedded Based Complete Vehicle Protection” April 2011

\section{AUTHORS}

Danish Karim is pursuing Masters of Technology at C-DAC Mohali in Embedded System. He has obtained his Bachelor of Engineering degree in Electronics \& Communication Engineering from Jai Prakash Univeristy, Chapra. His research interests include Embedded System.

Danish Karim is pursuing Masters of Technology at C-DAC Mohali in Embedded System. He has obtained his Bachelor of Engineering degree in Electronics \& Communication Engineering from Jai Prakash Univeristy, Chapra. His research interests include Embedded System.
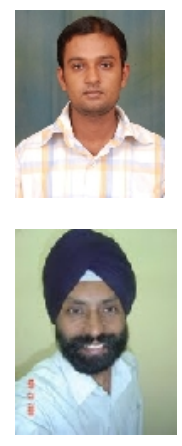